\title{
Magnetostriction offset of fluxgate sensors
}

\author{
Pavel Ripka, Mattia Butta and Michal Pribil \\ ${ }^{1}$ Faculty of Electrical Engineering, Czech Technical University in Prague, 16700 Czech Republic
}

\begin{abstract}
Magnetostriction is generally believed to cause excessive offset and noise in fluxgate sensors. We show that although the magnetostrictive core tapes are susceptible for offset instability, there is no simple direct mechanism for generation of $2^{\text {nd }}$ harmonic signal by magnetostriction. Offset and noise are caused by variation of local core properties and mechanical stresses together with magnetoelastic coupling.
\end{abstract}

Index Terms_-Fluxgate sensor, magnetic sensor, magnetostriction.

\section{INTRODUCTION}

$\mathrm{W}$ einer has declared in 1969 that magnetostriction causes excessive offset and noise in fluxgate sensors [1]. This paper has become classical and widely accepted [2]. Since that time near-zero-magnetostrictive alloys have been used for fluxgate cores and not much further attention was paid to this topic. In the first part of this paper we critically reexamine the theoretical description in [1] and show why we believe that it is not correct. According to our analysis magnetostriction itself without DC field component cannot cause the $2^{\text {nd }}$ harmonic signal and therefore the offset.

In the second section of this paper we experimentally investigate the correlation between magnetostriction of the core material and offset or noise of the fluxgate sensor. For this study we prepared a series of electrodeposited fluxgate cores with the same geometry and different magnetostriction.

We also describe experimental conditions, as both offset and noise strongly depend on the working point.

We believe that this study will help to understand problem which was marked as unsolved by Primdahl in 1979 [3].

\section{THEORY}

Classical paper of Weiner [1] influenced generations of researchers and fluxgate designers.

According to Weiner, periodic elongation of the sensor core caused by magnetostriction is a direct source of second harmonic. This signal cannot be distinguished from fielddependent signal and therefore causes sensor offset. The first reactions to Weiner's theory was negative: Gordon [4] attributed the dependence of noise on magnetostriction to indirect coupling of external stresses rather than by direct action of magnetostriction. Scouten [5] pointed out that magnetostriction should cause field-dependent signal rather than offset. This problem remained unsolved since then. Scouten has also shown using small search coils that sensor noise is a small-scale phenomenon compared to fluxgate mechanism. This indicates that the noise (and also the offset) is caused by random local isolated core volumes which are not saturated by the excitation field in particular magnetization cycle.

Narod [6] observed that minimum noise does not occur at

Manuscript received March 7, 2014. Corresponding author: P. Ripka (email: ripka@fel.cvut.cz

Digital Object Identifier inserted by IEEE alloys with zero magnetostriction, but at alloy with minimum core losses, which has both low saturation induction and coercivity. Nielsen [7] selected and processed the core material in order to minimize magnetostriction. However, even the finest fluxgate cores developed for Oersted satellite project have significant magnetostriction, as they emit audible acoustic noise.

First of all, we should highlight that the Weiner's paper is based on wrong formulas: in eq. 2 and 7 in [1], B and $\mathrm{H}$ should not be in absolute value. The first formula is derived from the induction law, which contains no absolute value. In the second case $\mathrm{dL}$ depends on $\mathrm{H}^{2}$, which means that $\mathrm{dL} / \mathrm{dH}$ should depend on $\mathrm{H}$, not $|\mathrm{H}|$. In fact Weiner apparently misinterpreted figures 5 and 6 (which were taken from Bozorth and already contain absolute values of dL/dH). As a result also eq. (8) in [1] is wrong.

Let us try to find offset sources related to global (i.e. constant in the whole volume of the core) magnetostriction. Offset is second harmonic component of voltage induced at the sensor output when the measured field $\mathrm{H}_{\mathrm{DC}}$ is zero.

Our description starts from the Faraday law. If we neglect the demagnetization effect, we can write for the induced voltage:

$$
\begin{array}{r}
V_{i}=-\frac{d \Phi}{d t}=\frac{d\left(N A \mu_{0} \mu_{r} H\right)}{d t} \\
=\mu_{0} N\left(H A \frac{d\left(\mu_{r}\right)}{d t}+H \mu_{r} \frac{d(A)}{d t}+A \mu_{r} \frac{d(H)}{d t}\right)
\end{array}
$$

where the magnetic flux $\Phi$ depends on the field $\mathrm{H}$, number of turns $\mathrm{N}$ and time-dependent permeability $\mu_{\mathrm{r}}(\mathrm{t})$, which is modulated by the excitation current. Here also A(t) is timedependent due to the magnetostriction.

The first term in (1) corresponds to the fluxgate effect, the second term corresponds to magnetostriction and third term corresponds to the induction effect. If the fluxgate has doublerod, racetrack or ring core, most of the signal is suppressed by symmetry; however this is irrelevant for the offset study, as the symmetry is never perfect.

The core field $\mathrm{H}$ has two components: $\mathrm{AC}$ excitation field $\mathrm{H}_{\mathrm{exc}}$ and DC measured field $\mathrm{H}_{\mathrm{DC}}$. If we search for magnetostrictive offset, we suppose that $\mathrm{H}_{\mathrm{DC}}=0$. We can also suppose that $\mathrm{H}=\mathrm{H}_{\mathrm{exc}}$ contains no even harmonics.

Let us examine the spectrum of $A(t)$. As $A(H)$ is even function, for odd $\mathrm{H}(\mathrm{t})$ then $\mathrm{A}(\mathrm{t})$ is even and also $\mathrm{dA} / \mathrm{dt}$ is even.

For the quantitative analysis we can use corrected Weiner's 
model [1]:

$\frac{1}{L} \frac{d L}{d H}=\frac{2 \lambda_{S}}{H_{S}^{2}} H$

If we neglect the volume magnetostriction we can write

$$
\frac{d A}{d t}=-\frac{d L}{d t}=-\frac{d L}{d H} \frac{d H}{d t}=-L \frac{2 \lambda_{s}}{H_{S}^{2}} H \frac{d H}{d t}
$$

If $\mathrm{H}(\mathrm{t})$ contains only odd harmonics $(\mathrm{H}(\mathrm{t})$ is "odd"), $\mathrm{dH} / \mathrm{dt}$ also contains only odd harmonics. The hysteresis loop is antisymmetric with respect to vertical axis, which means that $\mathrm{B}(\mathrm{H})$ is odd. On the contrary $\mu_{\mathrm{r}}(\mathrm{H})$ is even, so that for odd $\mathrm{H}(\mathrm{t})$ then $\mu_{\mathrm{r}}(\mathrm{t})$ is even.

The multiplication of two waveforms yields signal with a spectrum frequency components $f_{i j}=f_{i} \pm f_{j}$. If all $f_{i}$ and $f_{j}$ are even, than all $f_{i j}$ are even. If all $f_{i}$ and $f_{j}$ are odd, than all $f_{i j}$ are even. If all $f_{i}$ are even and all $f_{j}$ are odd, than all $f_{i j}$ are odd.

According to this rule $\mathrm{dA} / \mathrm{dt}$ contains only even harmonics. We can follow in similar way with the analysis of the other

TABLE I

HARMONIC COMPONENTS OF FLUXGATE SIGNALS

\begin{tabular}{l}
\multicolumn{3}{c}{ HARMONIC COMPONENTS OF FLUXGATE SIGNALS } \\
\begin{tabular}{|c|c|c|}
\hline \multicolumn{3}{|c|}{ EVEN OR ODD } \\
\hline $\mathrm{H}$ & odd & DC component \\
\hline $\mathrm{dH} / \mathrm{dt}$ & odd & 0 \\
\hline $\mathrm{dA} / \mathrm{dt}$ & even & 0 \\
\hline$\mu_{\mathrm{r}}$ & even & 0 \\
\hline 2nd term & odd & yes \\
\hline $\mathrm{A}$ & even & $\mathbf{0}$ \\
\hline $\mathrm{d} \mu_{\mathrm{r}} / \mathrm{dt}$ & even & yes \\
\hline 1st term & odd & 0 \\
\hline 3rd term & odd & $\mathbf{0}$ \\
\hline
\end{tabular}
\end{tabular}

components of (1) - the summarized results are shown in Table I.

\section{EXPERIMENTAL PART}

In this part we find indirect experimental proofs that the global magnetostriction does not cause offset.

For this study we prepared a series of electrodeposited 28 $\mathrm{mm}$ diameter Permalloy cores with the same thickness of $6 \mu \mathrm{m}$ and changing magnetostriction. The magnetostriction has been then measured using Becker-Kersten method from the dependence of saturation magnetization on applied stress: stress was induced by bending and magnetostriction was calculated as

$$
\lambda_{\mathrm{S}}=-1 / 3 \cdot \mu_{0} \cdot \mathrm{M}_{\mathrm{S}} \cdot \Delta \mathrm{H}_{\mathrm{S}} / \Delta \sigma
$$

where $\mathrm{M}_{\mathrm{S}}$ is saturation magnetization evaluated from the B$\mathrm{H}$ loop measured in the direction of applied stress.

Technological details of the core preparation and technique to measure magnetostriction are described in [8].

The core properties are summarized in Table II. Fig. 1 shows hysteresis curve of sensor \#104 without stress and with stress of $11.6 \mathrm{MPa}$ caused by bending. Each sensor had 270 turns of the excitation winding and 55 turns of the pick-up coil.

Typical sensor waveforms are shown in Fig. 2. All sensors were tuned by parallel capacitors both in the excitation and at the output. The excitation current was $25 \mathrm{kHz} / 800 \mathrm{~mA} \mathrm{p}-\mathrm{p}$, except for the measurement of the noise vs. excitation level.

The measurement of the sensor offset and noise was made in 6-layer permalloy shielding. The B-type uncertainty of this measurement is estimated to $1 \mathrm{nT}$, which is the residual field inside the shielding. Noise of fluxgate sensors is statistically unstable. In order to decrease the huge A-type uncertainty of the noise measurement we use overlapped averaging of noise spectra. Even though the A-type uncertainty of noise measurements is typically 10 to $20 \%$. By flipping the polarity of excitation and detector coils we are able to separate various parasitic offset components from the magnetic offset $\mathrm{B}_{0 \mathrm{mag}}$ [9]. Parasitic offset is caused by distortion in the excitation together with inductive and capacitive coupling $\left(\mathrm{B}_{\text {oind+cap }}\right)$ and also by the distortion in the processing electronics $\left(\mathrm{B}_{0 \mathrm{dis}}\right)$. When rotating the sensor core with respect to the pick-up coil, the magnetic offset changes. Fig. 3 shows typical angular dependence of $\mathrm{B}_{0 \mathrm{mag}}$ for cores with low and high magnetostriction. If the offset was caused by global property such as magnetostriction, it should be independent of the core rotation and appear as a shift of the curve. Such shift (average value) measured on a set of samples is shown in Fig. 4. The shift is very small and it has weak correlation with the magnetostriction.

TABLE II

PROPERTIES OF THE MEASURED CORES

\begin{tabular}{|c|c|c|c|c|c|}
\hline \hline \multicolumn{1}{c}{ Sample } & $\begin{array}{c}\lambda s \\
(\mathrm{ppm})\end{array}$ & $\begin{array}{c}\mathrm{H} \\
(\mathrm{A} / \mathrm{m})\end{array}$ & \multicolumn{1}{c}{$\begin{array}{c}\mathrm{Bm} \\
(\mathrm{T})\end{array}$} & \multicolumn{1}{c}{$\begin{array}{c}\mathrm{Br} \\
(\mathrm{T})\end{array}$} & $\mathrm{Br} / \mathrm{Bm}$ \\
\hline 111 & 9 & 97 & 0,68 & 0,45 & 0,67 \\
\hline 106 & 15 & 61 & 0,68 & 0,51 & 0,75 \\
\hline 108 & 35 & 95 & 0,83 & 0,56 & 0,67 \\
\hline 104 & 48 & 170 & 0,93 & 0,74 & 0,80 \\
\hline 107 & 55 & 140 & 0,92 & 0,71 & 0,77 \\
\hline 112 & 64 & 128 & 0,86 & 0,42 & 0,49 \\
\hline
\end{tabular}

Magnetic offset is standardly described as a core remanence. If the remanence is homogeneous, the angular dependence should be sinewave. Distortion shows that the remanence has significant local components for low-magnetostrictive alloy and global character for highly magnetostrictive alloy. Fig. 5 shows p-p value of the angular dependence of the offset. There is also correlation with magnetostriction, but we attribute it to the increasing coercivity and unidirectional stress combined with magnetostriction.

By integration (2) we get that below saturation the relative elongation $\lambda \approx \mathrm{H}^{2}$. If magnetostriction was a direct global source of offset, in this region the magnetostrictive offset and noise should be increasing with the excitation field amplitude. On the contrary, we and many others observed decrease of the offset with increasing excitation. Similar dependence is expected for the sensor noise. However, the noise level is monotonically decreasing with the excitation amplitude as shown in Fig. 6. The dependence between the magnetostriction and noise is shown in Fig. 7. Except for the last sample the correlation is clear.

\section{CONCLUSION}

Magnetostriction of otherwise ideal sensor core theoretically does not directly cause fluxgate offset. However, we observed 
an increase of the offset and a degradation of the offset stability and noise for sensor cores with large magnetostriction. We attribute this dependence to the coupling of magnetostriction with stress and temperature variations. Local variations of the core properties play crucial role: they can be caused by defects of many kinds and local mechanical stresses. Our study supports the traditional understanding of the offset caused by isolated core regions which have higher remanence.

\section{ACKNOWLEDGMENT}

This work has been supported by the Grant Agency of the Czech Republic (GACR) under the grant P102/12/2177.

\section{REFERENCES}

[1] M. Weiner, "Magnetostrictive offset and noise in flux gate magnetometers," IEEE Trans. Magn., vol. 5, pp. 98-105, Jun. 1969.

[2] P. Ripka, Magnetic sensors and magnetometers, Boston, London: Artech, 2001, pp. 88-90.

[3] F. Primdahl, "Fluxgate Magnetometer," J. Phys. E-Sci. Instrum., Vol. 12, pp. 241-253, Jan. 1979

[4] D. I. Gordon, R. H. Lundsten, and I. E. Scarzello, "Offset and noise in flux-gate magnetometers," IEEE Trans. Magn., vol. MAG-6, p. 818, Dec. 1970.

[5] D. C. Scouten, "Sensor noise in low-level fluxgate magnetometers," IEEE Trans. Mag., vol. MAG-8, pp. 223-231, June 1972.

[6] B. B. Narod, J. R. Bennest, J. O. Stromolsen,et al., "An evaluation of the noise performance of $\mathrm{Fe}, \mathrm{Co}, \mathrm{Si}$, and $\mathrm{B}$ amorphous-alloys in ring-core fluxgate magnetometers, "Canadian J. of Physics, vol. 63, pp. 14681472, Feb. 1985.

[7] O. V. Nielsen, J. R.Petersen, F. Primdahl, P. Brauer, B. Hernando, A Fernandez, J. M. G. Merayo, and P. Ripka, "Development, construction and analysis of the 'Orsted' fluxgate magnetometer," Meas. Sci. Technol., vol. 6, , pp. 1099-1115, June 1995.

[8] M. Butta, M. Janosek, P. Ripka, L. Kraus, and R. Kammouni, "The influence of magnetostriction of $\mathrm{NiFe}$ electroplated film on the noise of fluxgate," IEEE Trans. Magn., to be published.

[9] P. Ripka, M. Pribil, M. Butta, "Fluxgate offset study," IEEE Trans. Magn., to be published.

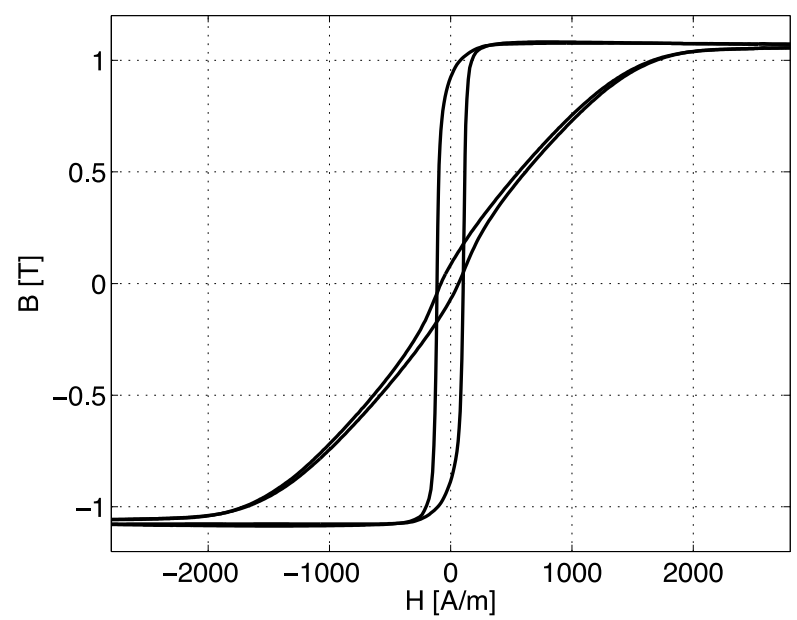

Fig. 1. Hysteresis loop of sensor \#104 without stress (soft curve) and with 11.6 MPa stress applied (hard curve).

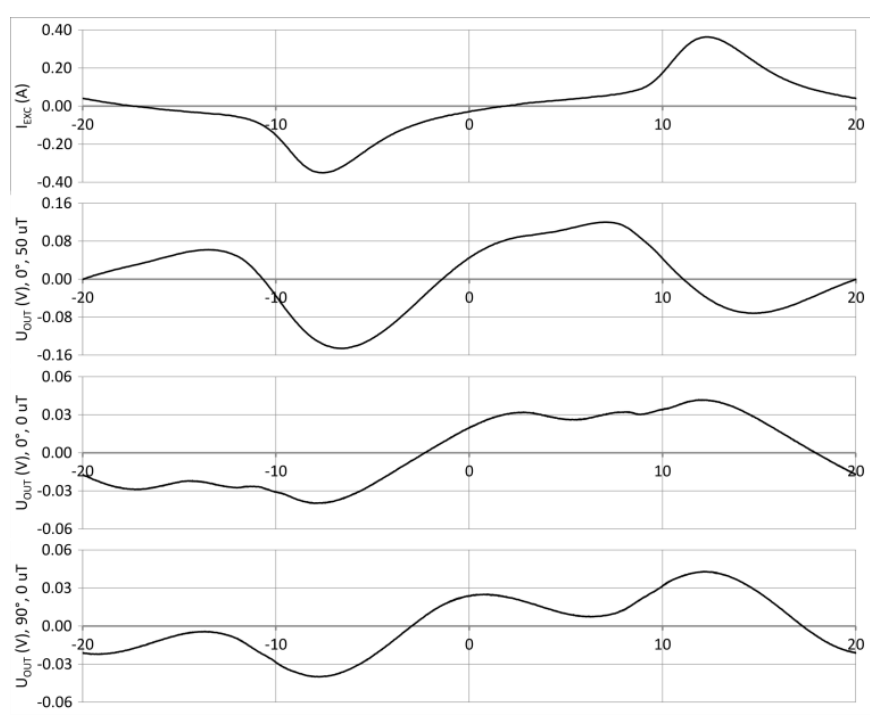

Fig. 2. Waveforms of tuned fluxgate: excitation current and output voltage for $\mathrm{B}=50 \mu \mathrm{T}, \mathrm{B}=0 \mathrm{~T}$ with $0^{\circ}$ core rotation corresponding to highest offset, and $\mathrm{B}$ $=0 \mathrm{~T}$ with $90^{\circ}$ core rotation - lowest offset
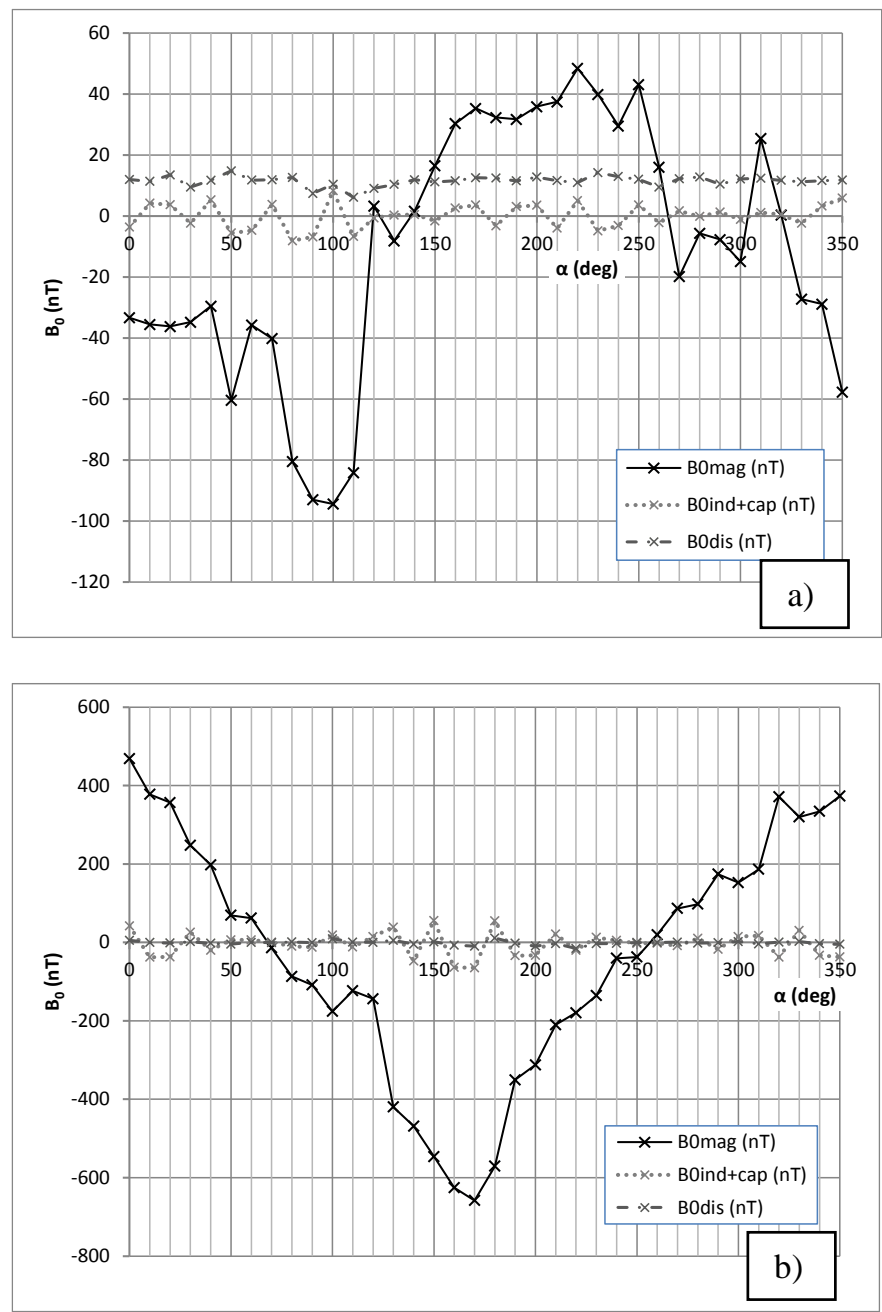

Fig. 3 Offset as a function of core rotation for a) low magnetostrictive $\left(\lambda_{S}=9\right.$ $\mathrm{ppm})$ and b) highly magnetostrictive $\left(\lambda_{\mathrm{S}}=64 \mathrm{ppm}\right)$ core material 


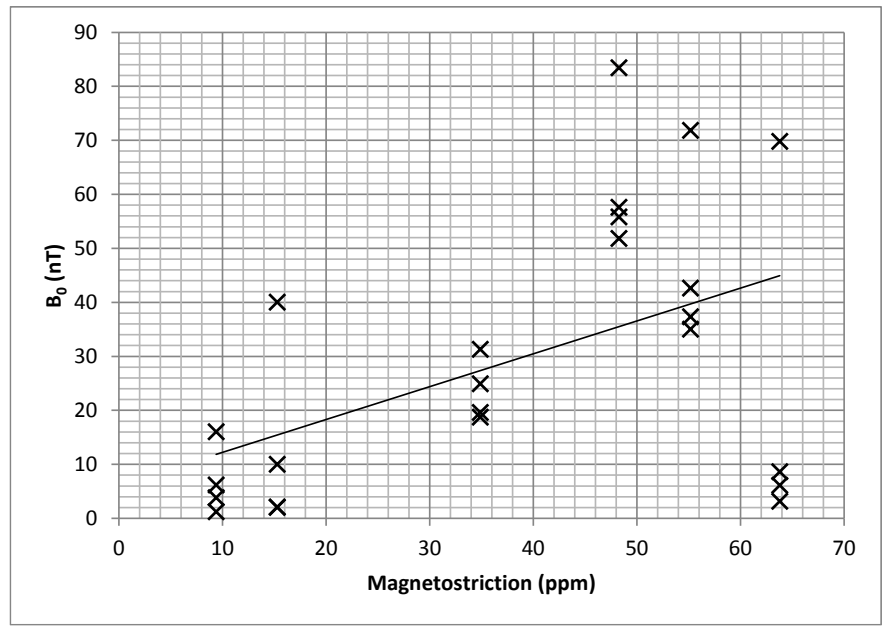

Fig. 4 Shift of the offset rotation characteristics. This represents a part of the offset which is independent of direction.

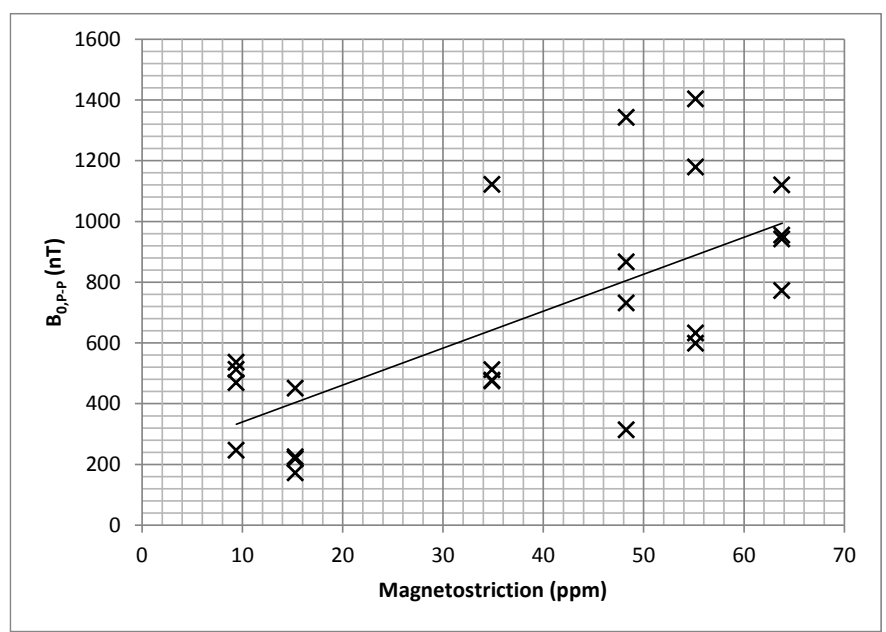

Fig. 5 p-p value of the offset rotation characteristics. This represents a part of the offset which is directionally dependent, such as global remanence

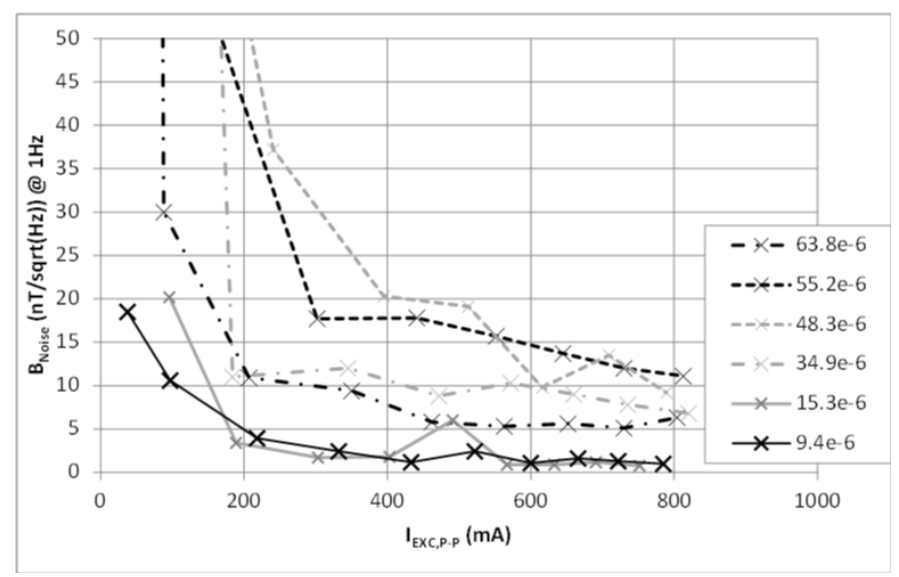

Fig. 6 Noise at $1 \mathrm{~Hz}$ as a function of the excitation amplitude for measured cores, magnetostriction is a parameter

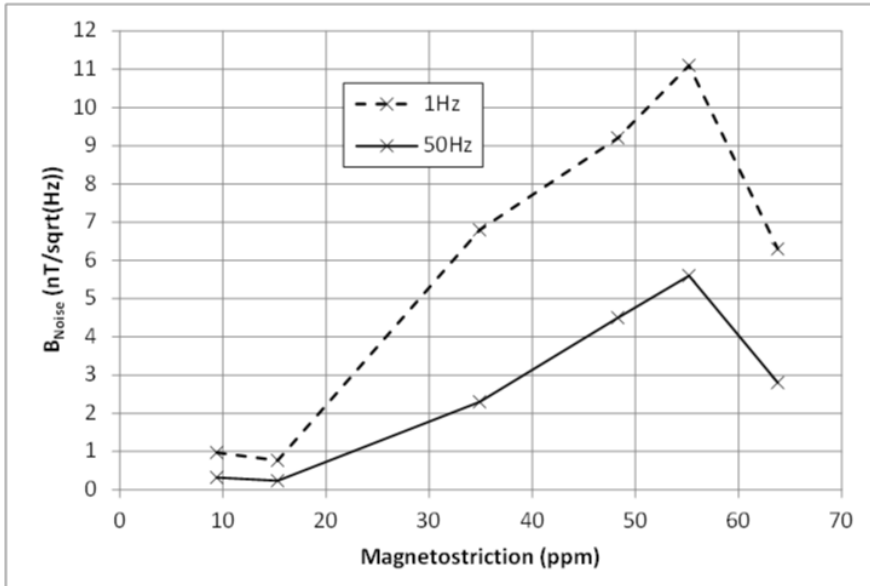

Fig. 7 Noise as a function of the core magnetostriction, measured for 800 mA p-p excitation. 